\title{
CD163 and its role in inflammation
}

\section{Krzysztof Kowal $^{1}$, Richard Silver ${ }^{2}$, Emila Sławińska ${ }^{3}$, Marek Bielecki ${ }^{4}$, Lech Chyczewski ${ }^{3}$, Otylia Kowal-Bielecka ${ }^{5}$}

\author{
${ }^{1}$ Department of Allergology and Internal Medicine, Medical University of Bialystok, Poland \\ ${ }^{2}$ Division of Rheumatology and Immunology, Medical University of South Carolina, \\ Charleston, SC, USA \\ ${ }^{3}$ Department of Medical Pathomorphology, Medical University of Bialystok, Poland \\ ${ }^{4}$ Department of Orthopedics and Traumatology, Medical University of Bialystok, Poland \\ ${ }^{5}$ Department of Rheumatology and Internal Medicine, Medical University of Bialystok, Poland
}

\begin{abstract}
Mononuclear phagocytes represent a heterogeneous population of cells with individual subpopulations exerting different pro- or anti-inflammatory functions. CD163 is a monocyte/macrophage specific marker expressed predominantly on cells which possess strong anti-inflammatory potential. The expression of CD163 is strongly induced by anti-inflammatory mediators such as glucocorticoids and interleukin-10, while being inhibited by pro-inflammatory mediators such as interferon-gamma. CD163-expressing mononuclear phagocytes, as well as soluble CD163, may both take part in downregulating an inflammatory response. It seems, therefore, that CD163 may be an interesting target for therapeutic modulation of the inflammatory response. (Folia Histochemica et Cytobiologica 2011, Vol. 49, No. 3, 365-374)
\end{abstract}

Key words: CD163, inflammation

\section{Introduction}

Monocytes and macrophages play an important role in the regulation of the inflammatory response $[1,2]$. Based on their functional properties, at least two subpopulations (M1 and M2) of mononuclear phagocytes, which differ in their pro- and anti-inflammatory potential, have been described [3]. The cells of the M1 subpopulation secrete predominantly pro-inflammatory mediators which trigger and amplify inflammatory responses. Those of the M2 subpopulation produce mainly anti-inflammatory mediators which take part in extinguishing inflammatory responses, but also play important roles in wound healing and angiogenesis [1-3]. Several sur-

Correspondence address: K. Kowal, Department of

Allergology and Internal Medicine,

Medical University of Bialystok,

M. Sklodowskiej-Curie Str. 24a, 15-276 Bialystok, Poland;

e-mail: kowalkmd@umwb.edu.pl face molecules have been used to identify those subpopulations. Recently, CD163 has been proposed to be a specific marker of monocytes/macrophages cell populations exhibiting strong anti-inflammatory properties [4]. Although CD163 is a marker of anti-inflammatory monocytes/macrophages, it also transduces signals upon binding of its ligands that lead to release of anti-inflammatory mediators such as interleukin-10 (IL-10).

CD163 is a type I transmembrane protein of 130 $\mathrm{kD}$ molecular weight belonging to the group $\mathrm{B}$ of the scavenger receptor cysteine-rich (SRCR) superfamily [4]. Dividing the SRCR superfamily receptors into two groups, A and B, depends on the number of cysteine residues present in each SRCR domain. Those cysteine residues participate in the formation of disulfide bonds which affect the folding and stability of the receptor. Each SRCR domain consists of 100-110 amino acids, and makes up part of the extracellular portion of the scavenger receptors belonging to that superfamily. Some differences in structure and 


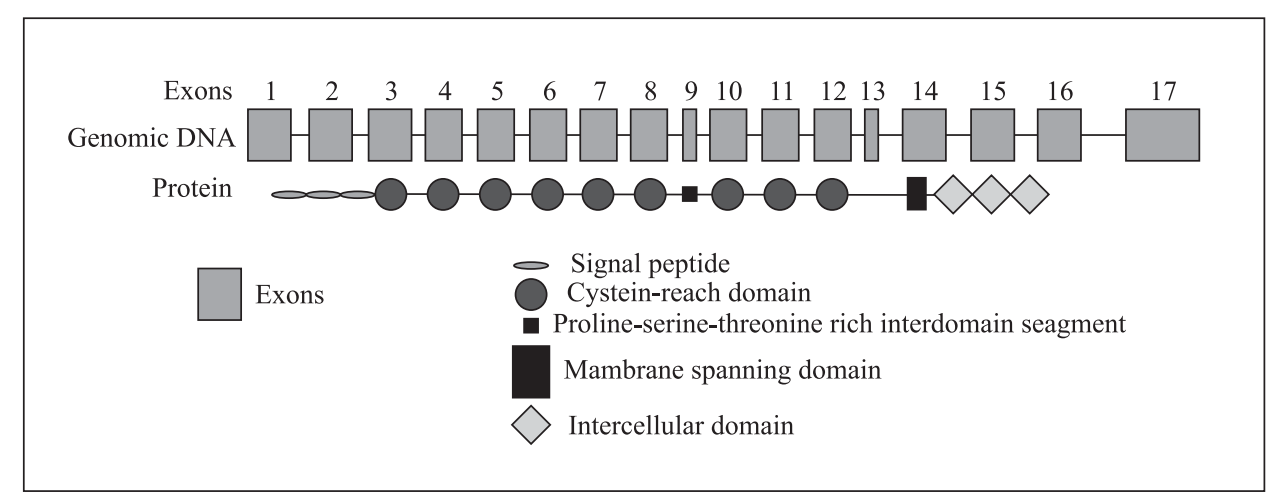

Figure 1. Schematic representation of genomic organization of CD163 gene in relation to individual structural parts of CD163 molecule

genetics of SRCR domains have been described. Group A SRCR domains contain six cysteines and are encoded by two exons, whereas Group B SRCR domains contain six or eight cysteines encoded by a single exon [5]. The members of class A and class B SRCR receptors contain several SRCR domains in their extracellular regions. Other molecules which belong to the class B SRCR superfamily, which share some homology with CD163, include CD5, CD6, $\mathrm{Sp} \alpha$, gp340/SAG/DMBT1, and the most closely related molecules CD163-L1, WC1 and SCART1.

There are nine SRCR class B domains in the extracellular part of CD163 (Figure 1). The sixth and seventh SRCR domains are separated by a short proline-serine-threonine rich interdomain segment encoded by a separate exon. The receptor also contains a transmembrane part spanning the plasma membrane once, and a short intracellular tail which contains consensus sequences for phosphorylation with protein kinase C and creatine kinase [4]. CD163 is heavily glycosylated with N-linked glycans. Treatment of CD163 with endoglycosidase F significantly reduces the molecular weight of CD163 [6].

Moreover, CD163 also exists as a soluble protein (sCD163) [4]. It is detectable in the plasma of healthy persons and can also be detected in body fluids such as synovial fluids [4]. It contains most of the extracellular part of the membrane CD163, including all SRCR domains [4].

\section{Gene structure}

The CD163 gene is located on the short arm of chromosome 12 (p13.31) [7]. A gene encoding CD163 molecule-like 1 is also located in the same region close to CD163. The CD163 molecule-like 1 (CD163-L1) gene is highly homologous to the CD163 gene and has the same orientation, suggesting that both genes might originate from a duplication of a single ances- tor gene. Other genes located in close proximity to CD163 include: complement component $1 \mathrm{~s}$ and $1 r$, tumor necrosis factor receptor-1, lymphotoxin-beta receptor, alpha2-macroglobulin, CD9, CD27, and $C D 69$ [7]. The CD163 gene spans more than $35 \mathrm{~kb}$ and consists of 17 exons and 16 introns $[7,8]$ (Figure 1). The first ATG codon is located in exon 1, and that exon also contains the $\mathrm{N}$-terminal part of the signal peptide. The remaining parts of the signal peptide are encoded by exon 2 and the first two nucleotides of exon 3. Exons 3 through 8 and exons 10 through 12 encode nine SRCR domains. Each exon encodes a separate SRCR domain. Exon 9 contains $93 \mathrm{bp}$ and encodes a short proline-serine-threonine rich interdomain segment of the receptor, which separates the sixth from the seventh SRCR domains. The 24 amino acid transmembrane part is encoded by a $72 \mathrm{bp}$ region within the exon 14. That exon also encodes the $\mathrm{C}$-terminal end of the extracellular part and the $\mathrm{N}$-terminal end of the intracellular part of CD163. The stop codon is located within exon 16, and exons 15 and 16 encode the $\mathrm{C}$-terminal part of the intracellular tail. The 3' untranslated region (3'UTR) is encoded by part of exon 16 and by exon $17[7,8]$.

The promoter region of human $C D 163$ lacks the canonical TATA consensus sequence, which is a feature of genes specifically expressed by myeloid cells $[8,9]$. Consistent with the absence of TATA-box, several transcription start sites have been described, with the major transcriptional start site being located $101 \mathrm{bp}$ upstream from the first ATG codon. In the promoter region, binding sites for transcription factors important for myeloid gene expression have been identified. Those include transcription factors of Ets-family (Ets and PU.1), Zn-finger family (Sp1), AP-1 family (AP-1) and C/EBP family $(\mathrm{C} / \mathrm{EBP} \alpha)$. Moreover, several putative binding sites for the glucocorticoid receptor and STAT-family transcription factors have also been demonstrated in the promoter region of the $C D 163$ gene $[8,9]$. 
Several alternative splicing variants of CD163 mRNA have been described. These include different splice variants in the 5' as well as in the 3' ends of the mRNA, and some of them may encode truncated CD163 protein $[7,8]$. No functional role for these individual CD163 splice variants has yet been described [7, 8].

Murine CD163 is highly homologous to human CD163 [10]. The gene encoding mouse CD163 is located on chromosome 6 in close proximity to complement components $1 \mathrm{r}$ and $1 \mathrm{~s}$ and to $C D 9$ [11]. That location is homologous to the gene cluster on human chromosome 12p13.3, which contains the human CD163 gene. Like human CD163, mouse CD163 is a type I transmembrane protein which contains a short (38-amino acid) signal peptide, nine SRCR domains, one transmembrane domain and a short cytoplasmic tail [10, 11]. High degrees of homology are also seen among swine, rat, monkey and human CD163 receptors [4].

\section{Expression of CD163 protein}

CD163 gene expression is tightly regulated in both a tissue-specific and a differentiation-specific manner $[8,12]$. It is detectable as both a cell surface receptor and also as a soluble form [13]. Cellular expression of CD163 is restricted to monocytes and macrophages $[8,12]$. Freshly isolated peripheral blood monocytes express a relatively low level of CD163, but this expression increases with differentiation of such cells into macrophages [14-17]. Original reports using Ber-Mac3 or RM3/1 antibodies demonstrated expression of CD163 on $5 \%$ or $30 \%$ of freshly isolated monocytes, respectively $[14,15]$. More recent reports, however, have demonstrated CD163 expression on a majority of peripheral blood monocytes $[16,17]$. The discrepancies in reported expression of CD163 on freshly isolated peripheral blood monocytes may be explained by the application of different antibodies and by different techniques used for monocyte isolation. Even the use of different anticoagulants in the cell selection process may result in differing intensity of CD163 staining [17]. Moreover, circulating monocytes represent heterogeneous cell populations with different expressions of CD163 on individual cell subpopulations. When monocytes are divided according to their expression of the endotoxin receptor (CD14) and the high affinity immunoglobulin $\mathrm{G}$ receptor type III (Fc $\gamma$ RIII - CD16), four different subpopulations can be distinguished $[18,19]$. The highest expression of CD163 is found on $\mathrm{CD} 14^{\text {high }} \mathrm{CD} 16^{+}$and the lowest on $\mathrm{CD} 14^{\text {low }} \mathrm{CD} 16^{-}$cells $[18,19]$. Cells in the $\mathrm{CD} 14^{\text {high }} \mathrm{CD} 16^{+}$subpopulation have been reported to exert predominantly anti-inflammatory functions. Most tissue macrophages, with the exception of lymphoid follicle macrophages, express CD163 on their surfaces and the expression is particularly high on liver Kupffer cells and on peritoneal and alveolar macrophages [12, 20]. The level of expression of CD163 on tissue macrophages has been shown to increase during the resolution of acute inflammatory response or during the wound healing phase [21, 22]. Neither granulocytes nor dendritic cells express significant levels of CD163. Expression of CD163 also depends on the maturation stage of monocytes/macrophages. To date, only one monocytic cell line, SU-DHL-1, has been shown to express CD163 [18]. Other mouse or human monocytic cell lines, including THP-1, U937, HL-60, P338, MonoMac6, RAW264.7 and J-774A.1, express no detectable CD163 at the mRNA level, either spontaneously or upon stimulation with phorbol esters (PMA) [18]. Glucocorticoid treatment, however, can induce CD163 expression on some of these cell lines, e.g. HL-60 [18]. Many pro- and anti-inflammatory mediators strongly affect CD163 expression (Table 1) [14-16, 18, 20, 22-33]. Expression of CD163 is strongly up-regulated by anti-inflammatory mediators including glucocorticoids and interleukin-10 (IL-10) [16, 18, 23-26]. In fact, in experiments utilizing gene-chip technology, CD163 displays the strongest response to IL-10 among all 19 of the up-regulated genes [25]. Interleukin 10 is also responsible for up-regulation of monocyte/macrophage CD163 expression induced by $\mathrm{CD} 4{ }^{+} \mathrm{CD} 25^{+}$Foxp $3^{+} \mathrm{T}$ regulatory cells and for rebound up-regulation of monocyte/macrophage CD163 expression after shedding of the receptor in response to Toll like receptor (TLR) stimulation $[27,28]$. Glucocorticoids are at least equally potent inducers of CD163 expression [16, 26]. The magnitude of up-regulation of CD163 expression depends on the potency of the glucocorticoid: those having the greatest affinity for the glucocorticoid receptor are the most potent for up-regulating CD163 expression [26]. Concomitant application to macrophages cultured in vitro of dexamethasone and IL-10 exerts an additive effect on CD163 expression [16]. Endogenous pro-inflammatory cytokines such as tumor necrosis factor-alpha (TNF- $\alpha$ ), IL-1 $\alpha$, IL- $1 \beta$ or chemokines such as CXCL-8 (IL-8) decrease expression of CD163 [16]. Similarly, exogenous pro-inflammatory molecules such as lipopolysaccharide (LPS) also decrease expression of CD163 [28, 29]. Interestingly, not only a Th- 1 type cytokine (interferon-gamma; IFN- $\gamma$ ) but also Th-2 type cytokines (IL-4 and IL-13) down-regulate CD163 expression [16]. These cytokines can suppress not only up-regulation of CD163 expression associated with differentiation of monocytes to mac- 
Table 1. Factors involved in regulation of CD163 expression

\begin{tabular}{|l|l|}
\hline $\begin{array}{l}\text { Factors up-regulating expression of CD163 } \\
\text { on monocytes/macrophages }\end{array}$ & $\begin{array}{l}\text { Factors down-regulating expression } \\
\text { of CD163 on monocytes/macrophages }\end{array}$ \\
\hline Glucocorticoids & Tumor necrosis factor-alpha (TNF- $\alpha$ ) \\
\hline Interleukin-10 & Interleukin-1 alpha (IL-1 $\alpha$ ) \\
\hline Interleukin-6 & Interleukin-1 beta (IL-1 $\beta$ ) \\
\hline Macrophage colony stimulating factor (M-CSF) & Interleukin-4 \\
\hline & Interleukin-13 \\
\hline & CCL-3 (MIP-1a) \\
\hline & CXCL-4 \\
\hline & CXCL-8 (IL-8) \\
\hline & Interferon-gamma (IFN- $\gamma$ ) \\
\hline & Transforming growth factor-beta (TGF- $\beta$ ) \\
\hline & Granulocyte macrophage colony stimulating factor (GM-CSF) \\
\hline & Lipopolysaccharide (LPS) \\
\hline & Stimulation of Toll-like receptors TLR-2, TLR-4, TLR-5* \\
\hline & Oxidative stress \\
\hline & Hypoxia \\
\hline & Cross-linking of Fcgamma receptor (Fc $\gamma$ R) \\
\hline & 8-iso-prostaglandin F2 $\alpha$ \\
\hline
\end{tabular}

*Prolonged stimulation with TLR agonists, however, leads to a rebound up-regulation of CD163 to levels higher than those observed before stimulation with TLR ligands through induction of IL-10 and Il-6

rophages, but also dampen IL-10- or glucocorticoid-induced CD163 expression [16]. Surprisingly, transforming growth factor-beta (TGF- $\beta$ ), which is an anti-inflammatory mediator, inhibits CD163 expression [30]. Growth factors which induce differentiation of monocytes into macrophages, such as macrophage colony stimulating factor (M-CSF), enhance CD163 expression, while those inducing differentiation of monocytes to dendritic cells, such as granulocyte macrophage colony stimulating factor (GM-CSF), down-regulate expression of CD163. Interleukin-6, which exerts pro- and anti-inflammatory effects on mononuclear phagocytes, enhances expression of CD163 [18].

Several chemokines have also been reported to affect CD163 expression on mononuclear phagocytes [16, 31]. CCL-3 (MIP-1a) and CXCL-8 down-regulate spontaneous and glucocorticoid-induced CD163 expression on cultured human monocytes [16]. Monocytes cultured in vitro in the presence of CXCL-4 express less CD163 than those cultured without that chemokine. Moreover, CXCL-4 prevented up-regulation of CD163 expression by M-CSF [31].

Activation of cell membrane Toll-like receptors (TLRs), such as TLR-2, TLR-4 or TLR-5, leads to a rapid down-regulation of CD163 expression by monocytes/macrophage cells [28]. This down-regulation of CD163 expression is caused by shedding of CD163 from the cell surface, which has been shown to involve a metalloproteinase-dependent process $[29,32]$. The same mechanism is responsible for down-regulation of monocyte CD163 expression by oxidative stress or 8-iso-prostaglandin F2 $\alpha$ [33]. Prolonged stimulation with cell membrane TLR agonists, however, leads to a rebound up-regulation of CD163 to levels higher than those observed before stimulation with TLR ligands [28]. This effect depends on delayed release of IL- 6 and IL- 10 by mononuclear phagocytes in response to TLR activation. When IL-6 and IL-10 are blocked, no rebound up-regulation of CD163 expression is seen [28]. Classical activation of monocytes/macrophages with IFN- $\gamma$ prevents up-regulation of CD163 in response to prolonged activation with TLR agonists [28]. Activation of intracellular TLRs does not, however, affect CD163 expression. Other factors which down-regulate CD163 expression include hypoxia and crosslinking of Fcgamma receptor $(\mathrm{Fc} \gamma \mathrm{R})[34,35]$. Hypoxia leads to inhibition of CD163 at the transcriptional level, while cross-linking of $\mathrm{Fc} \gamma \mathrm{R}$ results in shedding of CD163 from the cell surface [34, 35]. Immunoglobulin $\mathrm{G}$ immobilized on a culture plate, or complexes of $\mathrm{IgG}$ with an antigen, induce CD163 
shedding. This phenomenon depends on the presence of native $\mathrm{IgG}$, since denaturing of $\mathrm{IgG}$ totally aborts the shedding [35]. Interestingly, in contrast to prolonged TLR stimulation which results in IL-10-dependent up-regulation of CD163 expression, long-term stimulation of $\mathrm{Fc} \gamma \mathrm{R}$ does not cause rebound up-regulation of CD163 expression on mononuclear phagocytes [35].

CD163 also exists as a soluble protein (sCD163) detectable in the plasma of healthy humans [13]. The median serum concentration seen in healthy subjects is approximately $1.9 \mathrm{mg} / \mathrm{L}$ [13]. Plasma concentrations vary, particularly in patients with inflammatory diseases such as rheumatoid arthritis, asthma, multiple sclerosis, or celiac disease [36-39]. Elevated plasma/ /serum concentrations of sCD163 have also been reported in infectious diseases such as pneumonia, sepsis, meningitis or tuberculosis, in myeloid leukaemias, in atherosclerosis and in Gaucher disease which is characterized by the accumulation of tissue macrophages [13, 40-44]. Moreover, detectable levels of sCD163 in body fluids such as the synovial fluid of patients with rheumatoid arthritis or with spondyloarthropathy have been reported $[36,45]$. The main mechanism responsible for the appearance of sCD163 in body fluids is thought to be shedding of CD163 from the cell surface of mononuclear phagocytes $[29,32]$. At least two enzymes have been implicated in this process: matrix metalloproteinase-9 (MMP-9) [29] and tumor necrosis factor alpha-converting enzyme (TACE/ADAM17) [32]. An association between plasma MMP-9 levels and plasma sCD163 concentrations has been demonstrated in multiple sclerosis patients, supporting a role of MMP-9 in the regulation of CD163 shedding in vivo [38]. Several alternative splice variants of CD163 mRNA, which code for truncated forms of CD163 protein, have been reported. The transcriptional and/or translational regulation of CD163 expression does not seem to be the main mechanism affecting sCD163 levels.

Intravenous administration of hydrocortisone to healthy volunteers resulted in enhanced CD163 mRNA and protein expression on peripheral blood monocytes [46, 47]. Elevated numbers of CD163+ monocytes have been seen as soon as six hours after intravenous administration of glucocorticoids, and lasted for at least four days [46]. The maximum effect was seen between 12 and 24 hours after administration of glucocorticoids when more than $80 \%$ of monocytes stain positive with anti-CD163 antibody [46]. Applying different doses of a glucocorticoid has demonstrated that the peak effect is much less dose-dependent than are the rapidity and duration of the response [46].

\section{Function in inflammation}

Since its first description in 1987, CD163 (RM3/1) has been associated with the down-regulatory phase of the inflammatory process [22]. However, until 2001, an understanding of the biological role of CD163 was hampered by the fact that no naturally occurring ligand of CD163 was known and, therefore, the mechanisms of its anti-inflammatory action remained elusive [4]. The application of monoclonal antibodies that cross-link CD163, such as RM3/1, causes cell activation and the subsequent release of pro-inflammatory cytokines, including IL-6 and GM-CSF [48]. This effect, however, does not seem to be responsible for the main anti-inflammatory effects of CD163. One of the anti-CD163 antibodies, Ki-m8, possesses agonistic properties, and it has been shown that application of this antibody leads to induction of IL-10 secretion by mononuclear phagocytes [49].

In 2001, Kristiansen et al. demonstrated that CD163 binds to hemoglobin-haptoglobin (Hb-Hp) complexes, resulting in internalization of the complexes and preventing oxidative stress and inflammation associated with extracellular metabolism of $\mathrm{Hb}$ [50]. Using a ligand-affinity approach, CD163 was shown to be a specific receptor for Hb-Hp complexes, but not for $\mathrm{Hb}$ or $\mathrm{Hp}$ alone [50]. When released from erythrocytes, $\mathrm{Hb}$ binds to $\mathrm{Hp}$ to form complexes containing at least two $\mathrm{Hb}$ molecules. Formation of these complexes results in exposure of a new epitope, which allows binding to CD163 [50,51]. The binding site for $\mathrm{Hb}-\mathrm{Hp}$ complexes is located within the $3 \mathrm{SRCR}$ domain, and the binding process is both a calciumand $\mathrm{pH}$-dependent phenomenon [50,51]. When calcium concentration is below $0.5 \mathrm{mM}$, or $\mathrm{pH}$ is below 6.5, rapid dissociation of $\mathrm{Hb}-\mathrm{Hp}$ from CD163 occurs $[50,51]$. Binding of $\mathrm{Hb}-\mathrm{Hp}$ to $\mathrm{CD} 163$ activates the intracellular signaling which triggers release of IL-10 [49]. Subsequently the Hb-Hp-CD163 complex is internalized and $\mathrm{Hb}-\mathrm{Hp}$ is delivered to early endosomes, where CD163 dissociates and recycles to the plasma membrane, while $\mathrm{Hb}-\mathrm{Hp}$ undergoes intracellular metabolism within the lysosomes [52]. The heme undergoes intracellular degradation by the rate-limiting enzyme heme oxygenase-1 (HO-1), giving rise to free iron, biliverdine and carbon oxide (CO) [49, 53]. Altogether, the interaction of Hb-Hp with CD163 leads to release of IL-10 and CO, which exert strong anti-inflammatory effects. The release of IL-10 and $\mathrm{CO}$ by macrophages in response to binding of $\mathrm{Hb}-$ -Hp complexes has been demonstrated in vitro and in vivo [49]. Moreover, IL-10 acting in an autocrine and/ /or paracrine manner further up-regulates CD163 and HO-1 expression, creating a positive feed-back loop 
involved in efficient clearance of $\mathrm{Hb}$ and protection against triggering of an inflammatory response by extracellular $\mathrm{Hb}$. Therefore, protection against $\mathrm{Hb}-$ -induced inflammatory response conferred by CD163 is associated not only with scavenging of $\mathrm{Hb}-\mathrm{Hp}$ complexes from the extracellular milieu but also with active release of anti-inflammatory mediators such as IL-10 or CO (Figure 2).

Recently several other ligands of CD163, which play a role in the inflammatory response, have been described [54-58]. Screening of a combinatorial peptide library designed to search for a tumor necrosis factor-like weak inducer of apoptosis (TWEAK, TNFS12) ligands led to the discovery of motifs similar to those present in CD163 [54]. The discovery was reinforced by direct protein-protein interaction experiments, which showed that TWEAK specifically binds to CD163 [54]. TWEAK is a member of the TNF superfamily and plays many important roles in the processes associated with tissue damage and repair, e.g. inflammation, proliferation, apoptosis and angiogenesis [59]. Its ability to regulate innate and adaptive immune response seems to be one of the most interesting aspects of TWEAK from an immunological point of view [60]. In mice with genetically silenced TWEAK (TWEAK -/-), systemic administration of LPS results in a stronger inflammatory response with greater production of IFN- $\gamma$ and IL-12, but with less IL-10 than in wild-type mice [60]. This is associated with the development of more robust Th-1 type immune response [60]. In fact, TWEAK-/ /- mice are more resistant to the development of neoplasms whose growth is controlled by a Th- 1 type immune response [60]. Similarly, neutralization of TWEAK by monoclonal antibodies resulted in augmented production of IFN- $\gamma$ and IL-12 by human peripheral blood mononuclear cells cultured in the presence of LPS [60]. The major TWEAK receptor, fibroblast growth factor-inducible 14 (Fn14), is responsible for the signal transduction and cellular effects of TWEAK [59]. Many cells, including fibroblasts, endothelial cells and epithelial cells, release a broad range of chemokines, pro-inflammatory cytokines and matrix metalloproteinases (MMPs) in response to stimulation by TWEAK [57]. Monocytes express little or no Fn14 [54, 59]. They do, however, bind specifically TWEAK, and CD163 is responsible for that binding [54]. Further experiments revealed that the TWEAK binding site on CD163 is located in the region responsible for binding of $\mathrm{Hb}-$ -Hp complexes [54]. Analysis of protein databases showed that TWEAK contains regions with high homology to $\mathrm{Hb}$ and $\mathrm{Hp}$ beta chains, which may explain some similarities of the interactions of those proteins to CD163 [54]. Functional studies have demonstrated that the interaction of TWEAK with CD163 leads to neutralization of both molecules, suggesting that CD163 may function as a 'decoy' re-

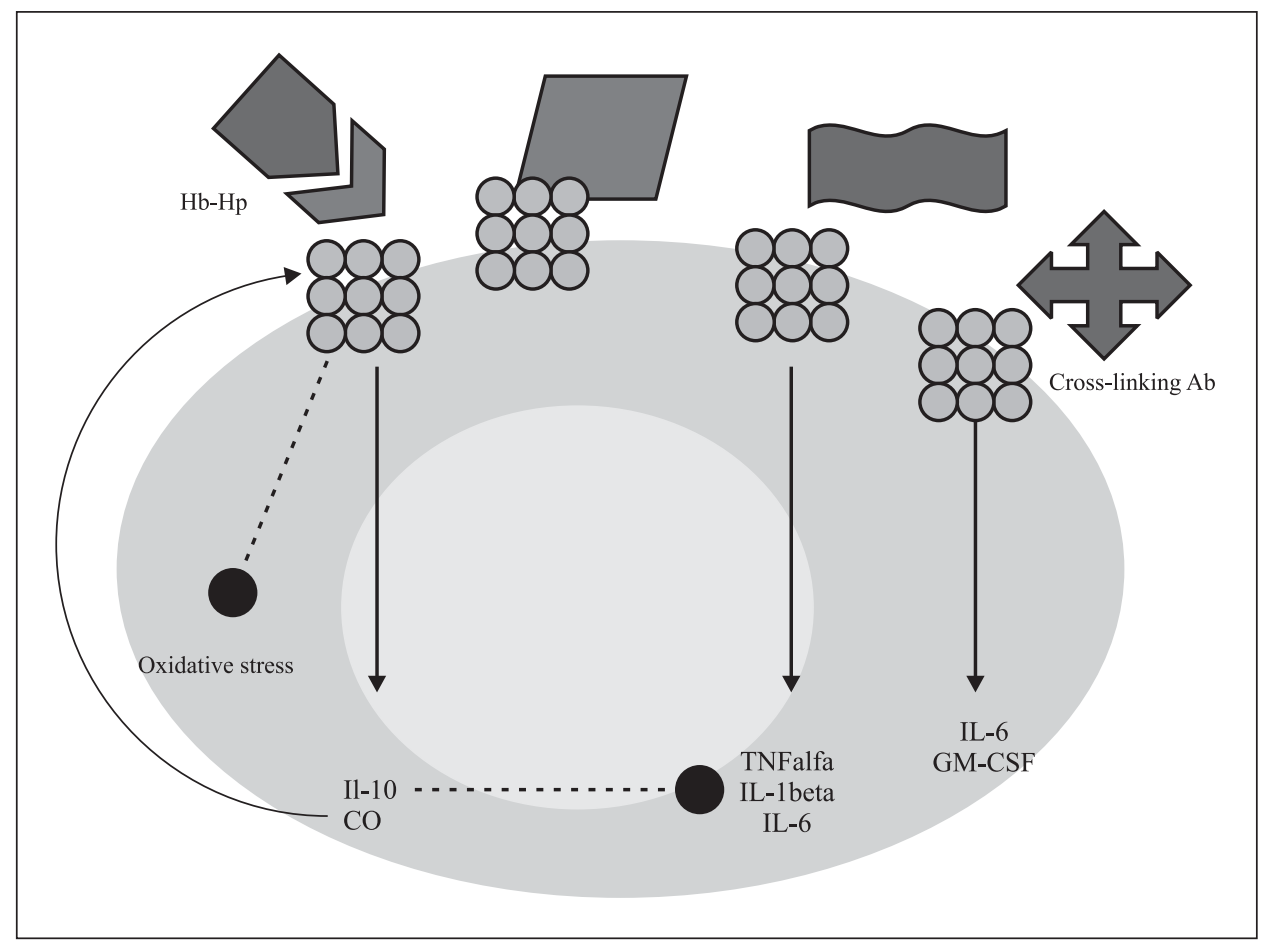

Figure 2. Schematic representation of anti-inflammatory effects mediated by CD163 
ceptor for TWEAK [54]. In fact, CD163 inhibits the apoptotic activity of TWEAK. On the other hand, in the presence of TWEAK, activation of monocytes by an anti-CD163 GHI/61 antibody is attenuated, indicating the presence of a common binding site on the CD163 molecule [54]. Indeed, high concentrations of sCD163 in plasma may be at least partially responsible for the lack of acute phase inflammatory response following systemic administration of TWEAK in mice [60]. Interestingly, IFN- $\gamma$ differentially regulates TWEAK and CD163 expression, inducing expression of the former and inhibiting expression of the latter $[18,59]$. Moreover, TWEAK impairs transition from innate to adaptive immunity which is associated with inhibition of IL-12 and IFN- $\gamma$ production [60]. Inhibition of TWEAK by CD163 may, therefore, skew the inflammatory response towards a Th-1 type immune response. On the other hand, TWEAK induces synthesis of many other pro-inflammatory mediators such as GM-CSF, IL-8 and RANTES, so inhibition of TWEAK by CD163 may in this way participate in extinguishing inflammation [59]. This may explain how during the inflammatory response, expression and function of those molecules may be tightly coordinated by proand anti-inflammatory cytokines.

CD163 binds not only to endogenous but also to exogenous ligands such as bacteria and viruses [56-58]. Gram-positive and gram-negative bacteria have each been shown to bind CD163 [56]. CD163 expressed on cell membrane as well as an immobilized protein efficiently binds bacteria. A peptide motif, GRIEIKFQGRW, located within the second SRCR domain of CD163, has been shown to be the major binding site [56]. Some binding ability by a peptide motif, GRLEVRFQGEW, in the third SRCR domain has also been demonstrated [56]. The binding by the latter motif was, however, consistently less efficient than that by the former one [56]. Interestingly, recognition of bacteria by CD163 results in amplification of pro-inflammatory cytokine production, including TNF- $\alpha$, IL- $1 \beta$ and IL- 6 , by mononuclear phagocytes, a response similar to that seen during TLR activation [56]. However, CD163, in contrast to TLRs, can recognize and bind intact bacteria and not only soluble microbial components [56].

In pigs, an important role of CD163 in viral infections has been demonstrated $[57,58]$. Two viruses, African swine fever virus (ASFV) and porcine reproductive and respiratory syndrome virus (PRRSV), use CD163 as an entry into the target cells of pigs $[57,58]$. The ASFV uses CD163 as a binding and internalization receptor, while for PPRSV, CD163 also plays a role in the uncoating process $[57,58]$. The susceptibility to infection for both viruses is proportional to the expression of CD163 on the cell surface $[57,58]$, and the viruses have a very restricted cell tropism for the monocyte/macrophage cells $[57,58,61]$. In a study of swine bone marrow monocyte precursors, peripheral blood monocytes and alveolar macrophages, the susceptibility of infection by ASFV was dependent on the degree of maturation of mononuclear phagocytes, being the lowest in bone marrow cells and the greatest in alveolar macrophages [57]. This was associated with differences in expression of CD163 on those various cells, being most abundantly expressed on alveolar macrophages [57]. Moreover, in vitro maturation of peripheral blood monocytes, which is associated with up-regulation of CD163, led to increased susceptibility of those in vitro matured cells to ASFV infection [57]. Although the natural host for the PPRSV is the pig, expression of dog, mouse, monkey or human CD163 can render cells at least partially permissive for PPRSV infection and replication [58, 61]. A series of mutation and genetic recombination studies have demonstrated that the fifth, but not the first, second or ninth, SRCR domain of CD163 is crucial for binding and internalization of PPRSV [62].

Altogether, studies strongly support the notion that membrane-bound CD163 functions as a receptor for endogenous and exogenous ligands, thereby participating in the initiation and/or perpetuation of the inflammatory response. Interestingly, the interaction of CD163 with some of its ligands results in a strong anti-inflammatory response, while other ligands trigger release of pro-inflammatory mediators. The particular response may at least partially depend on the location of the ligand binding site within the extracellular portion of CD163.

Not only does cell surface CD163 play a role in the modulation of the inflammatory response, soluble CD163 (sCD163) also can actively regulate that process. Soluble CD163 inhibits T cell proliferation $[63,64]$. Purified sCD163 inhibited in a dose-dependent manner phorbol ester-induced $\mathrm{T}$ cell proliferation in vitro, at an optimal concentration of sCD163 of $0.5 \mathrm{mcg} / \mathrm{ml}[63,64]$. This anti-proliferatory function of CD163 seems to be restricted to its soluble form, as membrane-bound CD163 does not exert such an effect [64]. The interaction between sCD163 and $\mathrm{T}$ lymphocytes is mediated by binding of sCD163 to non-muscle myosin heavy chain in T lymphocytes [65]. The associations between $\mathrm{T}$ cell proliferation and CD163 also come from in vivo studies in which elevated tissue expression of sCD163 is found in places where $\mathrm{T}$ cell proliferation is attenuated [66]. In par- 
ticular, in patients with rheumatoid arthritis, histological analysis of the inflamed joints revealed that wherever intense $\mathrm{T}$ cell proliferation was observed, little or no CD163 was seen on tissue macrophages [66]. Similarly, in asthmatic patients who develop prolonged airway inflammation associated with activation of allergen specific $T$ cells, decreased expression of monocyte CD163 and lack of increase in plasma sCD163 concentrations were demonstrated. On the contrary, asthmatic patients who did not develop late asthmatic response after allergen challenge were characterized by elevated concentrations of plasma sCD163 and an increase in monocyte CD163 expression [37]. Resolution of inflammatory response after allergen exposure is, therefore, associated with high plasma sCD163 concentrations, which is in line with original descriptions of elevated CD163 expression during resolution of inflammation. These findings may indicate that in allergic asthmatics CD163 is involved in the regulation of inflammatory response to allergen challenge. In chronic obstructive pulmonary disease (COPD), substantial expression of CD163 has been demonstrated on macrophages derived from bronchoalveolar lavage (BAL) or induced sputum [67]. Smoking cessation leads polarization of macrophages towards an anti-inflammatory phenotype, which is reflected by a higher percentage of macrophages expressing CD163 in BAL derived from ex-smokers. This study clearly demonstrates that exposure to tobacco smoke, an exogenous pro-inflammatory factor, is associated with downregulation of CD163 on BAL macrophages skewing the balance towards ongoing inflammatory response.

A recent study by Higashi-Kuwata et al. revealed increased expression of CD163 on skin macrophages and peripheral blood monocytes from patients with systemic sclerosis (scleroderma), an autoimmune disease characterized by local inflammatory infiltrates and widespread fibrosis [68]. The role of increased CD 163 in the pathogenesis of systemic sclerosis remains to be established.

\section{Perspectives}

Evaluation of CD163 expression would appear to be an interesting avenue in the assessment of the anti-inflammatory function(s) of monocytes/macrophages. CD163 is expressed primarily by monocytes/macrophages; therefore, it might be regarded as a relatively specific marker of monocyte/macrophage function. Moreover, variable degrees of up-regulation of CD163 expression by glucocorticoids may explain, in part, the anti-inflammatory effects of different glucocorticoids in vivo. It seems likely that evaluation of
CD163 expression in tissues or body fluids may also be a useful marker of local anti-inflammatory effects of glucocorticoids. Further studies are needed to confirm such an approach. The CD163 molecule might also prove to be an interesting therapeutic target. It is strongly up-regulated by anti-inflammatory mediators, e.g. glucocorticoids and IL-10, and both membrane-bound and soluble CD163 may at least partially mediate the anti-inflammatory effects of glucocorticoids. Therefore, one might speculate that application of sCD163 might be used for modulation of inflammatory responses in different clinical settings. A potential advantage of CD163 over glucocorticoids is that modulating CD163 might be devoid of the metabolic effects that are common adverse effects associated with the use of glucocorticoids in treating various immune diseases. Moreover, CD163 is a molecule naturally occurring in the human organism and, therefore, application of that molecule should not be associated with induction of an immune response such as allergic response.

\section{Summary}

CD163 plays a role in the regulation of the immune response. It affects the innate immune response as well as modulating the development of the adaptive immune response. Assessment of CD163 expression in different diseases may prove to be useful for the evaluation of disease severity and prognosis. Moreover, it may help in evaluation of anti-inflammatory therapy such as glucocorticoid therapy. Finally, targeting CD163 may be an interesting therapeutic approach for the treatment of many inflammatory diseases.

\section{References}

1. Auffrey C, Sieweke MH, Geissmann F. Blood monocytes: development, heterogeneity, and relationship with dendritic cells. Ann Rev Immunol. 2009;27:669-692.

2. Geissmann F, Manz MG, Jung S, Sieweke MH, Merad M, Ley K. Development of monocytes, macrophages, and dendritic cells. Science. 2010;327:656-661.

3. Yona S, Yung S. Monocytes: subsets, origin, fate and function. Current Opin Hematol. 2010:17:53-59.

4. van Gorp H, Delputte PL, Nauwynck HJ. Scavenger receptor CD163, a Jack-of-all-trades and potential target for cell directed therapy. Mol Immunol. 2010; epub.

5. Sarrias MR, Gronlund J, Padilla O, Madsen J, Holmskov U, Lozano F. The scavenger receptor cysteines-rich (SRCR) domain: an ancient and highly conserved protein module of the innate immune system. Crit Rev Immunol. 2004;24:1-37.

6. Fabriek BO, Polfliet MM, Vioet RP et al. The macrophage CD163 surface glycoprotein is an erythroblast adhesion receptor. Blood. 2007;109:5223-5229.

7. Ritter M, Buechler C, Langmann T, Schmitz G. Genomic organization and chromosomal localization of the human CD163 (M130) gene: a member of the scavenger receptor 
cysteine-rich superfamily. Biochem Biophys Res Comm. 1999; 260:466-474.

8. Law SKA, Micklem KJ, Shaw JM et al. A new macrophage differentiation antigen which is a member of the scavenger receptor superfamily. Eur J Immunol. 1993;23:2320-2325.

9. Ritter M, Buechler C, Langmann T, Orso E, Klucken J, Schmitz G. The scavenger receptor CD163: regulation, promoter structure and genomic organization. Pathobiology. 1999;67:257-261.

10. Schaer DJ, Boretti FS, Hongegger A et al. Molecular cloning and characterization of the mouse CD163 homologue, a highly glucocorticoid-inducible member of the scavenger receptor cysteines-rich family. Immunogenetics. 2001;53:170-177.

11. Schaer DJ, Schoeden G, Schaffner A. Assignment of the CD163 antigen (Cd163) to mouse chromosome 6 band F2 by radiation hybrid mapping. Cytogenet Genome Res. 2002; 98:231.

12. Radzun HJ, Kriepe H, Bodewadt S, Hansmann ML, Barth J, Parwaresch MR. Ki-M8 monoclonal antibody reactive with and intracytoplasmic antigen of monocyte/macrophage lineage. Blood. 1987;69:1320-1327.

13. Moller HJ, Peterslund NA, Graversen JH, Moestrup SK. Identification of the hemoglobin scavenger receptor/CD163 as a natural soluble protein in plasma. Blood. 2002;99:378-380 .

14. Backe E, Schwarting R, Gerdes J, Ernst M, Stein H. Ber-Mac3: a new monoclonal antibody that defines human monocyte/macrophage differentiation antigen. J Clin Pathol. 1991;44:936-945.

15. Hogger P, Dreier J, Droste A, Buck F, Sorg C. Identification of the integral membrane protein RM3/1 on human monocytes as a glucocorticoid-inducible member of the scavenger receptor cysteines-rich family (CD163). J Immunol. 1998;161:1883-1890.

16. Sulahian TH, Hogger P, Wahner AE et al. Human monocytes express CD163, which is upregulated by IL-10 and identical to p155. Cytokine. 2000;12:1312-1321.

17. Moniuszko M, Kowal K, Rusak M, Pietruczuk M, Dabrowska M, Bodzenta-Lukaszyk A. Monocyte CD163 and CD36 expression in human whole blood and isolated mononuclear cell samples: influence of different anticoagulants. Clin Vaccin Immunol. 2006;13:704-707.

18. Buechler C, Ritter M, Orso E, Langmann T, Klucken J, Schmitz G. Regulation of scavenger receptor CD163 expression in human monocytes and macrophages by pro- and anti-inflammatory stimuli. J Leukoc Biol. 2000;67:97-103.

19. Moniuszko M, Bodzenta-Lukaszyk A, Kowal K, Lenczewska D, Dabrowska M. Enhanced frequencies of $\mathrm{CD} 14^{++} \mathrm{CD} 16^{+}$, but not $\mathrm{CD} 14^{+} \mathrm{CD} 16^{+}$, peripheral blood monocytes in severe asthmatic patients. Clin Immunol. 2009;130:338-346.

20. Van den Heuvel MM, Tensen CP, van As JH et al. Regulation of CD163 on human macrophages: cross-linking of CD163 induces signaling and activation. J Leukoc Biol. 1999;66: 858-866.

21. Topoll HH, Zwadlo G, Lange DE, Sorg C. Phenotypic dynamics of macrophage subpopulations during experimental gingivitis. J Peridont Res. 1989;24:106-112.

22. Zwadlo G, Voegeli R, Schultze, Osthoff K, Sorg C. A mAb to a novel differentiation antigen on human macrophages associated with the down-regulatory phase of the inflammatory process. Exp Cell Biol. 1987;55:295-304.

23. Schaer DJ, Boretti FS, Schoedon G, Schaffner A. Induction of the CD163-dependent haemoglobin uptake by macrophages as a novel anti-inflammatory action of glucocorticoids. Br J Haematol. 2002;119:239-243.
24. Varga G, Ehrchen J, Tsianakas A et al. Glucocorticoids induce an activated, anti-inflammatory monocyte subset in mice that resembles myeloid-derived suppressor cells. $J$ Leukoc Biol. 2008;84:644-650.

25. Williams L, Jarai G, Smith A, Finan P. IL-10 expressing profiling in human monocytes. J Lekoc Biol. 2002;72:800-809.

26. Hogger P, Erpenstein U, Rohdewald P, Sorg C. Biochemical characterization of a glucocorticoid-induced membrane protein (RM3/1) in human monocytes and its application as a model system for ranking glucocorticoid potency. Pharm Res. 1998;15:296-302.

27. Tiemessen MM, Jagger AL, Evans HG, van Herwijnen MJC, John S, Taams LS. CD4 ${ }^{+} \mathrm{CD} 25^{+} \mathrm{Foxp}^{+}$regulatory T cell induce alternative activation of human monocytes/macrophages. Proc Natl Acad Sci USA. 2007;104:19446-19451.

28. Weaver LK, Pioli PA, Wardwell K, Vogel SN, Guyre PM. Up-regulation of human monocyte CD163 upon activation of cell-surface Toll-like receptors. J Leukoc Biol. 2007;81:663-671.

29. Hintz KA, Rassias AJ, Wardwell K et al. Endotoxin induces rapid metalloproteinase-mediated shedding followed by up-regulation of the monocyte hemoglobin scavenger receptor CD163. J Leukoc Biol. 2002;72:711-717.

30. Gleissner CA, Shaked I, Erbel C, Bockler D, Katus HA, Ley $\mathrm{K}$. CXCL4 downregulates the atheroprotective hemoglobin receptor CD163 in human macrophages. Circ Res. 2010;106:203-211.

31. Pioli PA, Goonan KE, Wardwell K, Guyre PM. TGF-b regulation of human macrophage scavenger receptor CD163 is Smad3-dependent. J Leukoc Biol. 2004;76:500-508.

32. Etzerodt A, Maniecki MB, Moller K, Moller HJ, Moestrup SK. Tumor necrosis factor alpha - converting enzyme (TACE/ /ADAM17) mediates ectodomain shedding of the scavenger receptor CD163. J Leukoc Biol. 2010; epub.

33. Timmermann M, Hogger P. Oxidative stress and 8-iso-prostaglandin F2a induce ectodomain shedding of CD163 and release of tumor necrosis factor-a from human monocytes. Free Radic Biol Med. 2005;39:98-107.

34. Bosco MC, Puppo M, Santangelo C et al. Hypoxia modifies the transcriptome of primary human monocytes: modulation of novel immune-related genes and identification of CC-chemokine ligand 20 as a new hypoxia-inducible gene. J Immunol. 2006;177:1941-1955.

35. Sulahian TH, Pioli PA, Wardwell K, Guyre PM. Cross-linking of FcgammaR triggers shedding of the hemoglobin-haptoglobin scavenger receptor CD163. J Lekoc Biol. 2004;76: 271-277.

36. Matushita N, Kashiwagi M, Wait R et al. Elevated levels of soluble CD163 in sera and fluids from rheumatoid arthritis patients and inhibition of the shedding of CD163 by TIMP-3. Clin Exp Immunol. 2002;130:156-161.

37. Kowal K, Moller HJ, DuBuske LM, Moestrup SK, Bodzenta-Łukaszyk A. Differential expression of monocyte CD163 in single- and dual-asthmatic responders during allergen-induced bronchoconstriction. Clin Exp Allergy. 2006;36:1584-1591.

38. Fabriek BO, Moller HJ, Vloet RP et al. Proteolytic shedding of the macrophage scavenger receptor CD163 in multiple sclerosis. J Neuroimmunol. 2007;187:179-186.

39. Daly DA, Walsh C, Feighery C, O'Shea U, Jackson J, Whelan A. Serum levels of soluble CD163 correlate with the inflammatory process in coeliac disease. Aliment Pharmacol Ther. 2006;24:553-559.

40. Moller HJ, Moestrup SK, Weiss N et al. Macrophage serum markers in pneumococcal bacteriemia: prediction of survival by soluble CD163. Crit Care Med. 2006;34:2561$-2566$. 
41. Knudsen TB, Larsen K, Kristiansen TB et al. Diagnostic value of soluble CD163 serum levels in patients suspected of meningitis: comparison with CRP and procalcitonin. Scand J Infect Dis. 2007; 39:542-253.

42. Knudsen TB, Gustafson P, Kronborg G et al. Predictive value of soluble haemoglobin scavenger receptor CD163 serum levels for survival in verified tuberculosis patients. Clin $\mathrm{Mi}$ crobiol Infect. 2005;11:730-735.

43. Aristoteli LP, Moller HJ, Bailey B, Moestrup SK, Kritharides L. The monocytic lineage specific soluble CD163 is a plasma marker of coronary atherosclerosis. Atherosclerosis. 2006;184:342-347.

44. Moller HJ, de Fost M, Aerts H, Hollak C, Moestrup SK. Plasma level of the macrophage-derived soluble CD163 is increased and positively correlates with severity in Gaucher's disease. Eur J Haematol. 2004;72:135.

45. Baeten D, Moller HJ, Delanghe J, Veys EM, Moestrup SK, De Keyser F. Association of CD163 ${ }^{+}$macrophages and local production of soluble CD163 with decreased lymphocyte activation in spondyloarthropathy synovitis. Arthritis Rheum. 2004;50:1611-1623.

46. Zwadlo-Klarwasser G, Bent S, Haubeck HD, Sorg C, Schmutzler W. Glucocorticoid-induced appearance of the macrophage subtype RM3/1 in peripheral blood of man. Int Arch Allergy Appl Immunol. 1990;91:175-180.

47. Yaeger MP, Pioli PA, Wardwell $\mathrm{K}$ et al. In vivo exposure to high or low cortisol has biphasic effects on inflammatory response pathways of human monocytes. Anasth Analg. 2008; 107:1726.

48. van den Heuvel MM, Tensen CP, van As JH et al. Regulation of CD163 on human macrophages: cross-linking of CD163 induces signaling and activation. J Leukoc Biol. 1999;66:858-866.

49. Philipidis P, Mason JC, Evans BJ et al. Hemoglobin scavenger receptor CD163 mediates interleukin-10 release and heme oxygenase-1 synthesis: anti-inflammatory monocyte-macrophage responses in vitro, in resolving skin blisters in vivo, and after cardiopulmonary bypass surgery. Circ Res. 2004;94:119-126.

50. Kristiansen M Graversen JH, Jacobsen C et al. Identification of the haemoglobin scavenger receptor. Nature. 2001;409: 198-201.

51. Madsen M, Moller HJ, Nielsen MJ et al. Molecular characterization of the haptoglobin-hemoglobin receptor CD163. J Biol Chem. 2004;279:51561-51567.

52. Schaer CA, Schoedon G, Imhof A, Kurrer MD, Schaer DJ. Constitutive endocytosis of CD163 mediates hemoglobin-heme uptake and determines the noninflammatory and protective transcriptional response of macrophages. Circ Res. 2006;99:943-950.

53. Nielsen DP, Moller HJ, Moestrup SK. Hemoglobin and heme scavenger receptors. Antioxid Redox Signal. 2010;12:261-273.

54. Bover LC, Cardo-Vita M, Kuniyasu A et al. A previously unrecognized protein-protein interaction between TWEAK and CD163: potential biological implications. J Immunol. 2007;178:8183-8194.
55. Moreno JA, Munoz-Garcia B, Martin-Ventura JL et al. The CD163-expressing macrophages recognize and internalize TWEAK: potential consequences in atherosclerosis. Atherosclerosis. 2009;207:103-110.

56. Fabriek BO, van Bruggen R, Deng DM et al. The macrophage scavenger receptor CD163 functions as an innate immune sensor for bacteria. Blood. 2009;113:887-892 .

57. Sanchez-Torrez C, Gomez-Puertas P, Gomez-del-Moral M et al. Expression of porcine CD163 on monocytes/macrophages correlates with permissiveness to African swine fever infection. Arch Virol. 2003;148:2307-2323.

58. van Gorp H, van Breedam W, Delputte PL, Nauwynck HJ. Sialoadhesin and CD163 join forces during entry of the porcine reproductive and respiratory syndrome virus. J Gen $\mathrm{Vi-}$ rol. 2008;89:2943-2953.

59. Burkly LC, Michaelson JS, Hahm K, Jakubowski A, Zheng TS. TWEAKing tissue remodeling by a multifunctional cytokine: role of TWEAK/Fn14 pathway in health and disease. Cytokine. 2007;40:1-16.

60. Maecker H, Vorlolomeev E, Kischkel F et al. TWEAK attenuates the transition from innate to adaptive immunity. Cell. 2005;123:931-944.

61. Welch SKW, Calvert JG. A brief review of CD163 and its role in PPRSV infection. Virus Res. 2010; epub.

62. van Gorp $\mathrm{H}$, van Breedam W, van Doorsselaere J, Delputte PL, Nauwynck HJ. Identification of the CD163 protein domains involved in infection of the porcine reproductive and respiratory syndrome virus. $J$ Virol. 2010;84:3101-3105.

63. Hogger P, Sorg C. Soluble CD163 inhibits phorbol ester-induced lymphocyte proliferation. Biochem Biophys Res Commun. 2001;288:841-843.

64. Frings W, Dreier J, Sorg C. Only soluble form of the scavenger receptor CD163 acts inhibitory on phorbol ester-activated T-lymphocytes, whereas membrane-bound protein has no effect. FEBS Letters. 2002;526:93-96.

65. Timmermann M, Buck F, Sorg C, Hogger P. Interaction of soluble CD163 with activated T lymphocytes involves its association with non-muscle myosin heavy chain type A. Immunol Cell Biol. 2004;82:479-487.

66. Fonseca JE, Edwards JCW, Blades S, Goulding NJ. Macrophage subpopulations in rheumatoid synovium. Reduced CD163 expression in $\mathrm{CD}^{+}{ }^{+} \mathrm{T}$ lymphocyte-rich microenvironments. Arthritis Rheum. 2002;46:1210-1216.

67. Kunz LIZ, Lapperre TS, Snoeck-Stroband JB et al. Smoking status and anti-inflammatory macrophages in bronchoalveolar lavage and induced sputum in COPD. Respir Res. 2011;12:34-42.

68. Higashi-Kuwata N, Jinnin M, Makino T et al. Characterization of monocyte/macrophage subsets in the skin and peripheral blood derived from patients with systemic sclerosis. Arthritis Res Ther. 2010;12:R128. 